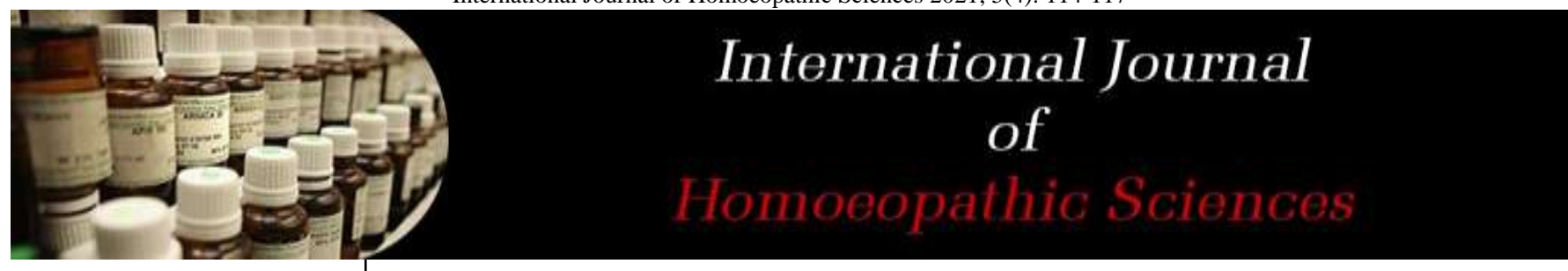

E-ISSN: 2616-4493 P-ISSN: 2616-4485 www.homoeopathicjournal.com IJHS 2021; 5(4): 114-117

Received: 11-08-2021

Accepted: 15-09-2021

Dr. Bed Prakash Gond Lecturer, Department of Community Medicine Netai Charan Chakravarty

Homoeopathic Medical College \& Hospital, (WBUHS),

Howrah, West Bengal, India
Corresponding Author: Dr. Bed Prakash Gond Lecturer, Department of Community Medicine Netai Charan Chakravarty Homoeopathic Medical College \& Hospital, (WBUHS), Howrah, West Bengal, India

\section{Black fungus/Mucormycosis \& its homoeopathic approach}

\section{Dr. Bed Prakash Gond}

DOI: https://doi.org/10.33545/26164485.2021.v5.i4b.459

\section{Abstract}

Mucormycosis is an angio-invasive infection that occurs due to the fungi mucorales. It is a rare disease but increasingly recognized in immuno-compromised patients. It can be categorized into rhinoorbitocerebral, pulmonary, cutaneous, gastrointestinal, disseminated, and miscellaneous types. The main aim and purpose of this review related to overview and Etiopathogenesis of Mucormycosis, clinical manifestations, recent advances in diagnostic and treatment methods and its Homoeopathic approach.

Keywords: Mucormycosis, fungal infection, diagnosis, zygomycosis, mucorales, diabetes mellitus, susceptibility, miasm, homoeopathy

\section{Introduction}

American pathologist R.D. Baker coined the term Mucormycosis. It represents a group of life-threatening infections caused by fungi of the order Mucorales of the subphylum Mucoromycotina (formerly known as the class Zygomycetes). Infection caused by the Mucorales is most accurately referred to as Mucormycosis, although the term Zygomycosis may still be used by some sources. Mucormycosis is highly invasive and relentlessly progressive, resulting in higher rates of morbidity and mortality than many other infections.

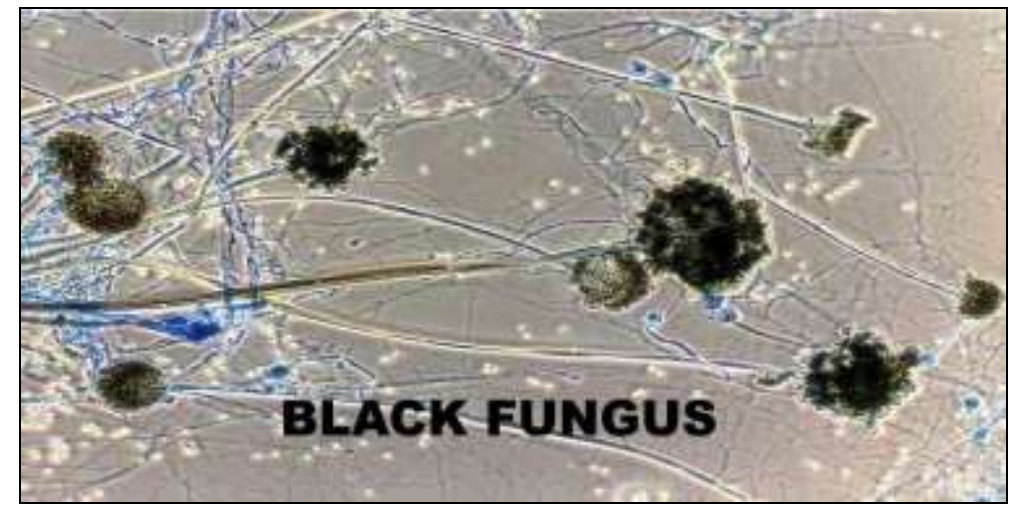

Fig 1: Microscopic view of showing dark spherical spores of black fungus reported in a case.

\section{Definition}

Mucormycosis is an acute fungal infection of nose and paranasal sinuses which may prove rapidly fatal. It is seen in uncontrolled diabetic or in those taking immunosuppressive drugs. From the nose and sinuses, infection can spread to orbit, cribriform plate, meninges and brain. Mucormycotina are the common saprobes originating from the rotten matter or soils. Infections with Mucorales are categorized by rapid progression.

\section{History}

In 1885, the German pathologist Paltauf, reported the first case of Mucormycosis and described it as Mycosis Mucorina. During 1980s and 1990s Mucormycosis was increasingly seen among immuno compromised individuals. Based on the prevalence rate, a study carried out in France reported amplification by $7.4 \%$ per year. 
Worldwide occurrence along with the possibility of seasonal variation of mucorales infection has been reported.

\section{Etiopathogenesis}

Mucorales attack deep tissues by means of ingestion or inhalation of spores, and percutaneous injection of spores. As soon as the spores penetrate into lung or cutaneous tissues, the first line of defence in the healthy host is capable of destroying the spores via oxidative metabolites and cationic peptides.

In diabetic patients, mucormycosis occurs as a destructive and potentially critical condition due to augmented availability of micronutrients and diminished defence mechanism of the body. Various hypotheses include (a) Low serum inhibitory activity against Rhizopus species, (b) Improved availability of iron for the pathogen at decreased PH level and (c) Pulmonary macrophages of persons with diabetes mellitus show diminished facility to inhibit germination of Rhizopus species. Ketone reductase in Rhizopus allows the organism to increase the glucose and acidic environment.

Neutrophils play a major role in host defence against mucorales. Its function is impaired at different level in DM. Ketoacidosis in diabetes accelerate the fungal invasion. The acidic milieu produces more free iron by reducing its binding to transferrin and low level of dialyzable inhibitory factor in diabetics present suitable conditions for fungal duplication. Mortality rate was reported $90 \%$ or even more with Mucormycosis.

Severely neutropenic patients and those who lack phagocytic function are more prone for mucormycosis. But it's not same in the case of AIDS patients. It implies that the $\mathrm{T}$ lymphocytes are not significant for inhibiting fungal proliferation but only the neutrophils. Moreover mucormycosis is also seen in patients without any obvious immune-deficiency. In such conditions, it may be related with burns, trauma and or allied with iatrogenic factors.

\section{Clinical Manifestations}

Infection of Mucormycosis in human beings occurs in two types: 1) Superficial and Visceral and 2) Localized and Disseminated. Superficial form occurs in external ear, fingernails, skin. Visceral forms are manifested as pulmonary, gastrointestinal and rhino cerebral types. Entry of these spores may takes place either through cutaneous or respiratory route. These categories of invasive mucormycosis tend to affect patients with specific defects in host defense.

\section{Rhino-orbital-cerebral}

Rhino-orbital-cerebral mucormycosis continues to be the most common form of the disease. The initial symptoms of rhino-orbital-cerebral mucormycosis are nonspecific and include eye or facial pain and facial numbness followed by the onset of conjunctival suffusion and blurry vision. Fever may be absent in up to half of cases. White blood cell counts are typically elevated as long as the patient has functioning bone marrow. If untreated, infection usually spreads from the ethmoid sinus to the orbit, resulting in compromise of extraocular muscle function and proptosis, typically with chemosis.

\section{Pulmonary}

Pulmonary mucormycosis is the second most common manifestation. Symptoms include dyspnoea, cough, and chest pain; fever is often but not invariably present. Angioinvasion results in necrosis, cavitations, and/or haemoptysis. Lobar consolidation, isolated masses, nodular disease, cavities, or wedge-shaped infarcts may be seen on chest radiography.

\section{Cutaneous}

Cutaneous mucormycosis may result from external implantation of the fungus or conversely from haematogenous dissemination. External implantation related infection has been described in the setting of soil exposure from trauma (For e.g., in a motor vehicle accident or natural disaster, penetrating injury with plant material, injection of medications).

\section{Differential Diagnosis}

Differential finding of mucormycosis include maxillary sinus neoplasia, maxillary sinus aspergillosis, soft tissue infarction, soft tissue radio necrosis, other deep fungal infections.

\section{Diagnosis}

Diagnosis of mucormycosis includes cautious evaluation of clinical manifestations, magnetic resonance imaging modalities, utilization of computed tomography (CT) in the early stages, specialist assessment of cytological and histological provision, and finest application of clinical microbiological technique and execution of molecular detection. Detection of host factors contributes extensively to the estimation of a patient's possibility for invasive mucormycosis. Histopathology culture examination can identify the Mucorales; specific species can be identified only by culture, while direct examination and Gomori methenamine silver stain, molecular methods and fluorescent in situ hybridization are the various laboratory techniques for detecting mucormycosis. Gold standard analytic technique for confirmation is the tissue based analysis.

\section{Treatment}

The successful treatment of mucormycosis requires four steps:

1. Early diagnosis.

2. Reversal of underlying predisposing risk factors, if possible.

3. Surgical debridement.

4. Prompt antifungal therapy.

Early diagnosis of mucormycosis is critical, since early initiation of therapy is associated with improved survival rates. It is also crucial to reverse (or prevent) underlying defects in host defense during treatment.

\section{General Management}

1. Monitor the sick person's symptoms regularly.

2. Ensure the sick person takes adequate rest and sleep and stays hydrated.

3. Limit the patient's movement around the home and minimize shared space. Ensure that the house, particularly shared spaces (e.g. kitchen, bathroom) are well ventilated and damp proof, with proper sunlight.

4. Maintain personal hygiene and use separate mask for each day. 


\section{Homoeopathic Approach on Mucormycosis}

Homoeopathy is one of the most popular holistic systems of medicine. The selection of remedy is based upon the theory of individualization and symptoms similarity by using holistic approach. The aim of Homoeopathy is not only to treat mucormycosis symptoms but to remove its underlying cause and individual susceptibility. Fungal infections are manageable to Homoeopathic treatment. Various research studies undertaken on various fungi model showed that Homoeopathy medicine could prevent the growth of the fungus and disease originated due to fungus.

\section{Miasmatic point of view}

With respect to the origin of the chronic maladies as in the acute, miasmatic eruptional diseases, three different important movements are to be more attentively considered than hitherto been done, says master Hahnemann.

i). Firstly, the time of infection.

ii). Secondly, the period of time during which the whole organism is being penetrated by the disease infused, until it has developed within.

iii). Thirdly, the breaking out of the external ailment.

Dr. Stuart close say's, the cause of disease is an infective agent which our master Hahnemann and most of his followers called as "MIASM". Miasms are dynamic disease producing powers which pollute the human organisms and produces all possible diseases. Dr. J.H. Allen, in his "Chronic miasm" explains that, "We cannot select the most similar remedy possible, unless we understand the phenomenon of the acting and basic miasm; for the true similia is always based on the existing basic Miasms, whether we are conscious or unconscious of the fact. However, the miasmatic cause of Mucormycosis is "Trimiasmatic or Mixed-miastic". Dr. H.A. Roberts says. "In treating the combined stigmata, the most outstanding must be treated first, since we base our method of treatment upon symptom similarity, where psora is present psora will be the most outstanding in the symptom totality in the earlier manifestations. These manifestations must be treated first, then after it is eradicated or considered lessened, the next most potent dyscracia, as it expresses itself in the symptomatology must be treated, until this way each time treating the most dominant stigma, as expressed by the outward manifestations, until cure is attained."

\section{Homoeopathic Therapeutics \\ Arsenicum Album}

1. Burning pain, the parts burn like fire.

2. Gangrenous aphthae, which burns like fire.

3. All the symptoms worse at night, particularly after midnight.

4. Great anguish, extreme restlessness, and fear of death.

5. Great thirst for cold water drinks very often, but takes but little at a time.

\section{Aurum Metallicum}

1. Through the organic nervous system it especially acts upon the palatine bones; osseous system, more especially the nasal and palatine bones.

2. Caries of the nasal, palatine, mastoid, and ossicular bone - RAUE.

3. Swelling of the skull bones.

4. Caries of bones, paining worse at night.
5. Aggravation in the morning; on getting cold; while reposing.

6. Relieved by moving; while walking, and on getting warm.

\section{Acidum Benzoicum}

1. Through the organic nervous system, it acts on the urinary organs, joints, fibrous tissue, and skin.

2. Especially adapted to rheumatic or gouty subjects, where the urine is excessively fetid.

3. The urine is high coloured, with an exceedingly strong smell; dark-coloured and much heavier than normal.

4. Concretions in the joints, with rheumatism, or gout, with strong-smelling urine.

5. Articular rheumatism, with fetid urine.

\section{Condurango}

1. Painful Cracks in Corner of Mouth is a guiding symptom of this drug. Chronic gastric catarrh, syphilis, and cancer. Tumors; stricture of oesophagus.

2. Fissures form about the muco-cutaneous outlets. Epithelioma of lips or anus. Ulcerative stage of carcinoma cutis when fissures form.

\section{Kali Bichromicum}

1. Acts through the ganglionic system upon the mucous membranes; the glandular system; fibrous tissue and skin.

2. The mucous membranes chiefly affected are the mouth, throat, cardiac portion of the stomach, duodenum, jejunum, and rectum;

3. The whole respiratory membrane, including the conjunctiva and the uterus.

4. Upon the skin it causes papules, pustules, and ulcers.

5. Upon the fibrous tissue, about the joints and the periosteum, it has a marked and powerful influence. It also affects the cartilages, especially that of the nose, which it has entirely destroyed.

\section{Secal Cornutum}

1. Strong tendency to putrescence, discharge of black blood; a kind of sanies, with tingling in the limbs and great debility.

2. Passive haemorrhages; everything seems open and loose; no action, in thin, scrawny, cachectic women.

3. Dry gangrene of the extremities; the parts are dry, cold, hard, and insensible, of a uniform black colour, and free from fetor - HEMPEL.

4. The ulcer feels as though it had been burnt; discharges a putrid, bloody fluid, and is sometimes decidedly gangrenous and painless; in thin, scrawny cachectic people.

\section{References}

1. Chander Jagdish. Textbook of Medical Mycology, Jaypee Brothers Medical Publishers (P) Ltd. ${ }^{\text {th }}$ Edition, 2020, Chapter 26 "MUCORMYCOSIS".

2. Kasper Daniel L. Harrison's Principle of Internal Medicine, $19^{\text {th }}$ Edition, Copyright (C) 2015 by McGrawHill Education, Section-16 "Fungal Infection" Chapter242 "Mucormycosis".

3. Information for Homoeopathic Practitioners for Symptomatic Management of Suspected and Diagnosed case of Mucormycosis, Drug Policy Section, Ministry 
of Ayush, Govt. of India.

4. Ramalingam Suganya, Mucormycosis: A Brief Review, Suganya et al. J Pure Appl Microbiol 2019;13(1):161165 | March Article 5470.

5. Allen JH. The Chronic Miasm, Bjain Publishers (P) LTD, reprint 2004.

6. Nagendra Babu G, Comprehensive Study of Organon, Bjain Publishers (P) LTD.

7. Dudgeon RE. Organon of Medicine, $5^{\text {th }} \& 6^{\text {th }}$ Edition, Indian Books \& Periodical Publishers.

8. Allen HC. Allen's Keynotes, Rearranged and Classified with Leading Remedies of the Materia Medica and Bowel Nosodes, 10 ${ }^{\text {th }}$ Edition, B. Jain Publishers Pvt. Ltd 2005.

9. Burt William H. Characteristic Materia Medica, University of Michigan Library 2006.

10. Phatak SR. Concise Materia Medica of Hom. Remedies, B Jain Publishers Pvt. Ltd; Enlarged Edition 2003.

11. Boericke William-Homoeopathic Materia Medica \& Repertory, B Jain Publishers Pvt. Ltd 2003.

12. Murphy ND. Robin-Lotus Materia Medica- B Jain Publishers Pvt. Ltd. 2004-2 ${ }^{\text {nd }}$ Edition. 